\title{
Vertebral Brown Tumor in Childhood (Case Report)
}

\author{
Csenge Szeverényi ${ }^{1}$, Balázs Dezső $^{2}$, Tamás Deményi ${ }^{3}$, Zoltán Csernátony $^{1}$ \\ ${ }^{1}$ Department of Orthopedic Surgery, Debrecen, Hungary \\ ${ }^{2}$ Department of Pathology, Debrecen, Hungary \\ ${ }^{3}$ University of Debrecen Medical and Health Science Center, General Practitioner, Debrecen, Hungary \\ E-mail: szcsenge@dote.hu \\ Received February 23, 2011; revised May 27, 2011; accepted June 9, 2011
}

\begin{abstract}
One form of renal osteodystrophy secondary to chronic renal failure is the osteitis fibrosa cystica, which is the complication of secondary hyperparathyroidism. Osteitis fibrosa cystica is a histologically benign, tumor-like lesion of bones. Since early diagnosis and treatment of hyperparathyroidism is available nowadays, the development of osteitis fibrosa cystica has become rare. It can only be found in neglected cases and in those where the treatment of chronic renal failure is unsuccessful. We present the case of a boy, who developed osteitis fibrosa cystica as a complication of secondary hyperparathyroidism. Our article is the first to report a case about an osteitis fibrosa cystica found in a child's vertebra and this tumor was the only one, which reached a size of approximately $10 \times 15 \times 15 \mathrm{~cm}$. We present the CT films and X-ray pictures, the surgical solution and the results of the histological examination.
\end{abstract}

Keywords: Vertebral Localization, Osteitis Fibrosa Cystica, Secondary Hyperparathyroidism, Chronic Renal Failure

\section{Introduction}

Brown tumor (i.e. osteitis fibrosa cystica, osteoclastoma, giant cell reparative granuloma) is a lytic bony lesion in non-treated or uncontrollable hyperparathyroidism. Osteitis fibrosa cystica may develop in primary and in secondary hyperparathyroidism as well. The most frequent cause of secondary hyperparathyroidism is chronic renal failure. Since the early diagnosis and treatment of hyperparathyroidism are available nowadays, the occurrence of brown tumor became very rare [1-7].

Our main objective with this case report is to draw attention to the possible occurrence of brown tumors in rare locations throughout the body and to provide an insight on the diverse manifestation and localizations of these lesions and the comprehensive treatment of this disease and its complications.

\section{Materials and Methods}

\section{Case Report}

The 9-year-old male was taken to the doctor because of enuresis. His renal failure was diagnosed at that time. The results of the kidney biopsy performed were unable to clarify the cause of the disease since it was in an ad- vanced stage. Histology revealed severe glomerular, nodular sclerosis. Conservative therapy (calcium carbonate, sodium bicarbonate) was started in order to treat the renal failure and it was recommended to keep a strict diet. However, regular haemodialysis became necessary two years later. Elevated parathyroid hormone levels were detected 6 months after diagnosing the child's renal failure \{PTH: 27.0 pmol/L (Reference range: 0.8 - $5.2 \mathrm{pmol} / \mathrm{L}$; Primary hyperparathyroidism: 45.0 - $58.6 \mathrm{pmol} / \mathrm{L}$; chronic renal failure: $0.9-418.0 \mathrm{pmol} / \mathrm{L}$ ) , which was the result of his chronic renal failure. Parathyroid scintigraphy was performed four years later because of his extremely high PTH levels. This study excluded the possibility of a parathyroid adenoma in the cervical region and in the mediastinum as well. Cervical CT scan revealed a persistent thymus and calcifications in the soft tissue in the retrosternal region and near the spinal processes of the vertebrae. Meanwhile, the patient was excluded from the waiting list of renal transplantation candidates, because of his severe obesity. Subtotal parathyroidectomy was performed four months later due to high PTH levels (PTH: 188.5 pmol/L). Histopathology of the parathyroid revealed hyperplasia. A few months later, the child was admitted to our Department, in order to remove the large $(15 \times 15 \times 10 \mathrm{~cm})$ tumor on the rear side of his neck 
(Figure 1). The abnormality was detected a half year before the operation for the first time and it was only $2 \times$ $1 \times 3 \mathrm{~cm}$ large at that time.

Chest CT scan verified calcifications in the soft tissue at the levels of the C7-Th4 vertebrae in the deep back muscles of the left paravertebral region, the destruction and calcification of the spinous process of the 7th cervical vertebra and a 10x15x15 cm large cystic tumor at the level of C1-Th2 vertebrae, whose caudal part was calcified (Figure 1).

During the surgery the cervical cyst was prepared and removed with multiple cysts and solid tumors spreading into the muscles of the back. There was no communication between the cyst and the liquor space, but the cyst contained thick, white-yellowish fluid (Figure 2).

\section{Results}

According to the histological findings, the removed tumor was diagnosed as osteitis fibrosa cystica, which was the complication of the chronic hyperparathyroidism (Figure 3).

On the basis of the imagery the tumor's origin was in the spinous process of the 7th cervical vertebra and starting from that location, it invaded the nearby soft tissues. Despite of the parathyroidectomy performed 4 months earlier, the child's PTH serum level remained high.

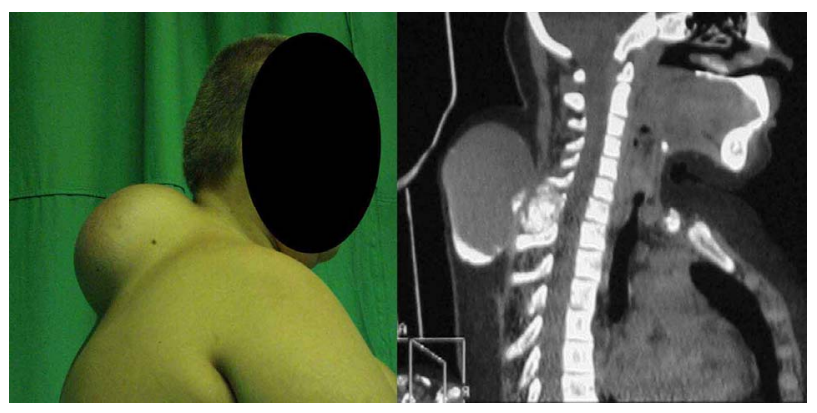

Figure 1. The tumor on the neck of the child grew to $15 \times 5$ $\times 10 \mathrm{~cm}$ large in half a year. On the chest CT scan image, the deformity and calcification of the spinous process of the 7 th cervical vertebra and the cystic tumor are visible.

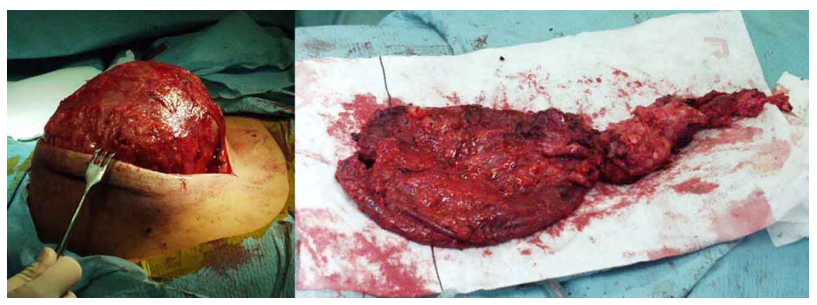

Figure 2. During the surgical intervention, the cervical cyst was removed with the other cystic and solid tumors spreading into the muscles of the back.

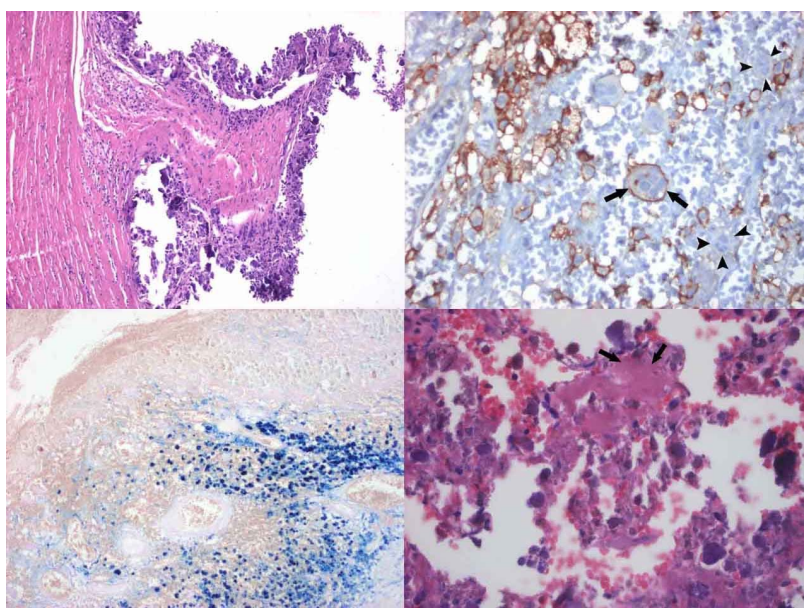

Figure 3. Upper left section: the histopathology of the hematoxylin-eosin (HE) stain tissue section clearly shows that the wall of the cyst is not covered by epithelium, therefore the cyst is actually a pseudocyst. The lumen is covered by necrotic debris with inflammatory cells, mainly macrophages (original magnification: 10x). Upper right section: immunohistochemistry (IHC) for CD68 identifies macrophages (cells with brown membrane staining) including foreign body giant macrophages (arrows). Note that osteoclast type of giant cells are CD68 negative (arrowheads). (IHC with hematoxylin nuclear counter staining; original magnification: 20x). Lower left section: Berlin blue staining reveals the accumulation of hemosiderin laden macrophages (original magnification: 10x). Lower right section: despite the huge size of the tumor, some remnant bone trabeculae (arrows) can be discovered in the histological sections. The big dark-blue (basophilic) precipitates correspond to dystrophic calcifications (original magnification: 40x).

\section{Discussion}

In view of the fact that in chronic renal failure the matrix of the kidneys shrinks, the production of the active vitamin D will decrease, which has a primary etiological role in the development of the brown tumor. Vitamin D facilitates the absorption of calcium from the intestines, and it inhibits phosphate reabsorption in the kidneys. Low levels of vitamin D lead to hypocalcaemia and phosphate retention, which stimulate parathyroid hormone secretion [4-6].

Parathyroid hormone facilitates the differentiation of osteoclasts and these cells increase the serum calcium level by bone resorption. In osteitis fibrosa cystica, reactive fibrosis of the bone marrow can also be detected in addition to the bone resorption caused by the activity of the osteoclasts. The focal bone resorption and the progression of the fibrosis result in macroscopically visible cysts, which build up the brown tumor [3,4,7-10]. During the tumor growth the bone may become weak resulting in pathologic fractures or spread into the soft tissues 
causing compressive symptoms $[4,6]$. Furthermore, as in our case, the tumor has a potential to become so large that it makes everyday life difficult.

PTH also has an effect on the kidneys as it increases phosphate excretion. Therefore, hypersecretion of PTH can be inhibited by eliminating phosphate excess. It can be achieved by oral antacids, dietetic restrictions and peritoneal dialysis or haemodialysis $[4,10,11,12]$. However, after a certain period of time, the enlarged parathyroid gland might not react to the fluctuant serum calcium level, therefore some degree of autonomous glandular function develops $[5,13]$. When this occurs, conservative treatment cannot prevent progressive bone resorption, hypercalcaemia and metastatic calcifications anymore. In these cases subtotal parathyroidectomy may be effective, although secondary hyperparathyroidism might recur, due to the presence of any residual parathyroid gland tissue [4]. In our case, even though parathyroidectomy was performed, serum PTH levels remained high (PTH: $206.1 \mathrm{pmol} / \mathrm{L}$ ). This arose the suspicion the presence of an autonomous functioning, ectopic parathyroid tissue in the persistent thymus or somewhere else in the body, causing hypersecretion of PTH.

Based on the literature reviewed, the incidence of brown tumor involving bones is between 1.5\% - 13\% among patients suffering from chronic renal failure [4,6, 7]. Furthermore, the lesion develops most frequently in the pelvic bones, costae, sternum, maxillary sinus, mandible and in the femur $[3,4,7,8,12,13]$. The presence of brown tumor in the vertebral column is rare. After reviewing the literature since 1974, only 19 similar cases were published, with 8 patients having primary and 11 having secondary hyperparathyroidism as the underlying cause of the disease $[1,4-7,12,14]$. We did not find a single case of brown tumor developing from a vertebra of a child and reaching such an enormous size. The significance of osteitis fibrosa cystica involving the vertebrae is that it may cause the pathologic fractures of the vertebrae or it may invade the spinal canal causing the compression of the spinal cord $[1,4-7,12,14]$.

\section{Conclusions}

Our publication focused on the diagnosis, adequate treatment and the prevention of possible complications of brown tumors, which manifested as a divers multi-local disease in our case. Brown tumor is a complication of chronic renal failure and even though brown tumor has become very rare nowadays, one has to think of it in patients suffering from chronic renal failure, and especially in those cases in which the metabolic balance cannot be achieved by conservative methods. In certain cases surgical excision or spine-stabilizing intervention may be- come necessary due to the localization or the size of the tumor. In our case, finding and removing the suspected ectopic parathyroid tissue and completing the kidney transplantation may bring significant improvement to the patient's state of health.

\section{References}

[1] S. D. Ashebu, M. H. Dahniya, S. A. Muhtaseb and P. Aduh, "Unusual Florid Skeletal Manifestations of Primary Hyperparathyroidism,” Skeletal Radiology, Vol. 31, No. 12, 2002, pp. 720-723. doi:10.1007/s00256-002-0578-9

[2] M. E. Bohlman, Y. C. Kim, J. Eagan and E. K. Spees, "Brown Tumor in Secondary Parathyroidism Causing Acute Paraplegia,” American Journal of Medicine, Vol. 81, No. 3, 1986, pp. 545-547. doi:10.1016/0002-9343(86)90312-8

[3] H. Dursun, O. Kucukosmanoglu, A. Noyan, N. Ozbarlas, M. Buyukcelik, M. Soran, A. K. Bayazit and A. Anarat, "Mitral Annular Calcification and Brown Tumor of the Rib in a Child with Chronic Renal Failure," Pediatric Nephrology, Vol. 20, No. 5, 2005, pp. 673-675. doi:10.1007/s00467-004-1721-8

[4] K. Fineman, P. Johnson, P. L. Di-Patre and H. Sandhu, "Chronic Renal Failure Causing Brown Tumors and Myelopathy. Case Report and Review of Pathophysiology and Treatment," Journal of Neurosurgery, Vol. 90, No. 4, 1999, pp. 242-246.

[5] A. O. Mustonen, M. J. Kiuru, A. Stahls, T. Bohling, A. Kivioja and S. K. Koskinen, "Radicular Lower Extremity Pain as the First Symptom of Primary Hyperparathyroidism," Skeletal Radiology, Vol. 33, No. 8, 2004, pp. 467-472.

[6] F. Tarrass, A. Ayad, M. Benjelloun, A. Anabi, B. Ramdani, M. G. Benghanem and D. Zaid, "Cauda Equina Compression Revealing Brown Tumor of the Spine in a Long-Term Hemodialysis Patient,” Joint Bone Spine, Vol. 73, No. 6, 2006, pp. 748-750. doi:10.1016/j.jbspin.2006.01.011

[7] E. Vandenbussche, L. Schmider, C. Mutschler, M. Man, C. Jacquot and B. Augereau, "Brown Tumor of the Spine and Progressive Paraplegia in a Hemodialysis Patient," Spine, Vol. 29, No. 12, 2004, pp. E251-E255. doi:10.1097/01.BRS.0000127187.58944.FA

[8] A. Dogan, E. Algun, E. Kisli, M. Harman, M. Kosem and N. Tosun, "Calcaneal Brown Tumor with Primary Hyperparathyroidism Caused by Parathyroid Carcinoma: An Atypical Localization," Journal of Foot and Ankle Surgery, Vol. 43, No. 4, 2004, pp. 248-251. doi:10.1053/j.jfas.2004.05.001

[9] F. Gouin, E. Grimaud, F. Redini, A. Moreau, N. Passuti and D. Heymann, "Metatarsal Giant Cell Tumors and Giant Cell Reparative Granuloma Are Similar Entities,” Clinical Orthopaedics and Related Research, Vol. 416, 2003, pp. 278-284. doi:10.1097/01.blo.0000093016.90435.f6 
[10] B. B. Pecovnik and R. Kavalar, "Brown Tumor in Association with Secondary Hyperparathyroidism. A Case Report and Review of the Literature," American Journal of Nephrology, Vol. 18, No. 5, 1998, pp. 460-463.

[11] S. Lee, D. B. Lerer, H. D. Dorfman and M. Coco, "Brown Tumors Developing in Renal Transplant Recipients with Persistent Hyperparathyroidism: Two Case Reports and Review of Literature," Clinical Nephrology, Vol. 61, No. 4, 2004, pp. 289-294.

[12] M. Marini, A. Vidiri, R. Guerrisi, F. Campodonico and R. Ponzio, "Progress of Brown Tumors in Patients with Chronic Renal Insufficiency Undergoing Dialysis,”
European Journal of Radiology, Vol. 14, No. 1, 1992, pp. 67-71. doi:10.1016/0720-048X(92)90066-I

[13] J. W. Milgram, C. A. Engh, C. R. Hamilton Jr. and G. M. Kammer, "Renal Resistance to Parathyroid Hormone with Hyperphosphatemic Osteomalacia and Osteitis Fibrosa. A Case Report," Journal of Bone and Joint Surgery, Vol. 56, No. 7, 1974, pp. 1493-1500.

[14] K. U. Lewandrowski, D. Togawa, T. W. Bauer and R. F. McLain, "A Role for Vertebral Biopsy in Selected Patients with Known Malignancy. A Report of Three Cases,” Journal of Bone and Joint Surgery, Vol. 87, No. 6, 2005, pp. 1348-1353. doi:10.2106/JBJS.C.01664 